\title{
OUTCOME OF CHILDREN AT 1-2 YEARS AND MATERNAL MORBIDITY AFTER CESAREAN SECTION VS VAGINAL BIRTH FOR BREECH PRESENTATION AT OR NEAR TERM
}

Jyoti Ramesh Chandran, Uma Devi. N, D. Sumangala Devi

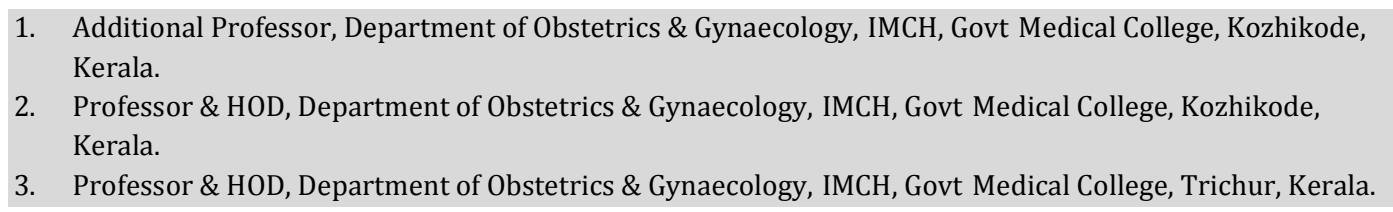

\section{CORRESPONDING AUTHOR}

Dr. Jyoti Ramesh Chandran,

Additional Prof Obst \& Gynae,

Chandra Villa, Behind KSEB Chungam,

PO: West Hill,

Kozhikode, Kerala- 673005.

E-mail :drjyotichandran@gmail.com

Ph: 00919995353512

ABSTRACT: Back ground: In 2000 an international multicentric randomized control trial of planned vaginal delivery vs planned elective caesarean section for uncomplicated term breech presentation confirmed that perinatal mortality and serious neonatal morbidity were significantly lower in planned caesarean group. Secondary analysis of Term Breech Trial showed that prelabour caesarean and caesarean during early labour were associated with lowest adverse perinatal outcome due to labour or delivery and that vaginal delivery had the highest risk of adverse outcome AIMS: The purpose of this study is to determine the outcome of children at 1-2 years and maternal morbidity after caesarean section vs. vaginal birth for breech presentation at or near term. SETTINGS AND DESIGN: Prospective observational study from $1^{\text {st }}$ January 2008-30th June 2009( 18 months ) at Institute of Maternal \& Child Health , Govt. Medical College Kozhikode. METHODS AND MATERIAL: Outcome of 45 assisted breech deliveries during this period analyzed from case records. 90 Cesarean deliveries during the same period randomly selected as control. STATISTICAL ANALYSIS USED: Data analyzed using SPSS version 16.0.Chi square test was used to compare the outcome. A p value $<0.05$ was considered to indicate statistical significance. RESULTS: Vaginal delivery group- Five minute Apgar $<7$ ( $p=0.019)$, NICU admission ( $\mathrm{p}=0.00001)$ (RR 4.71, 95\% CI 2.33 to 9.91),Neonatal morbidity ( $\mathrm{p}=0.012) \mathrm{RR} 2.627,95 \%$ CI 1.216 to 5.678 ,Prolonged hospitalisation $(\mathrm{p}=0.005) \mathrm{RR}=$ $2.962,95 \%$ CI 1.354 to 6.478 statistically significant in vaginal delivery group .Caesarean Section group-Elective 30(33.3\%) Emergency 60(66.6\%) Neonatal complication( $\mathrm{p}=0.03$ ) $\mathrm{RR}=2.57,95 \%$ CI 1.06 to 6.2 , NICU admission ( $p=0.013 \mathrm{RR}=2.86,95 \%$ CI 1.21 to 6.76. were statistically significant in elective section group Maternal morbidity was not associated with type of CS p=0.2 RR=1.39,95\% CI 0.447 to 4.307 . However the perinatal mortality was not significantly different in either group $p=0.167$ (RR 2.67,95\%CI 0.62 to 11.41) CONCLUSION:There is an inherent risk of increased immediate neonatal morbidity with vaginal breech delivery. But at 1-2 year follow up the maternal and infant outcome is not significantly different in the two groups.

KEYWORDS: vaginal breech delivery 
INTRODUCTION: In 2000 an international multicentric randomized control trial of planned vaginal delivery vs planned elective caesarean section for uncomplicated term breech presentation confirmed that perinatal mortality and serious neonatal morbidity were significantly lower in planned caesarean group. Secondary analysis of Term Breech Trial showed that prelabour caesarean and caesarean during early labour were associated with lowest adverse perinatal outcome due to labour or delivery and that vaginal delivery had the highest risk of adverse outcome

SETTINGS AND DESIGN: This was a prospective study from $1^{\text {st }}$ January 2008-30 th June 2009 (18 months) at Institute of Maternal \& Child Health, Govt. Medical College Kozhikode.

STATISTICAL ANALYSIS USED: Data analyzed using SPSS version 16.0. Chi square test was used to compare the outcome. A p value $<0.05$ was considered to indicate statistical significance.

METHODS AND MATERIAL: Outcome of 45 assisted breech deliveries during this period analyzed from case records and followed up for 1-2 years. 90 Cesarean deliveries during the same period randomly selected as control.

RESULTS AND ANALYSIS: The two groups were comparable in terms of maternal age, parity, gestational age and neonatal birth weight.

Table 1 shows that $62.2 \%$ of patients who underwent C-section and $64.4 \%$ delivered vaginally were in the age group between $19-25$ years. $72.2 \%$ of primigravida underwent Csection and $62.2 \%$ delivered vaginally. $77.8 \%$ were at gestational age $37-40$ weeks. Of those who underwent C-section for breech 33.3\% were elective and 66.6 emergency.88.8\% of those who had C-section and 93.3\% who delivered vaginally had babies weighing 1500-3500 gm. . Babies with weight $>3500$ were delivered by C-section only $(7.8 \%) 6.7 \%$ of babies with vaginal delivery had Apgar $<7$ at 5 minutes ( $\mathrm{p}=0.019$ ). $51.1 \%$ of babies delivered vaginally needed NICU admission (RR 4.71, 95\% CI 2.33 to 9.91) ( $\mathrm{p}=0.00001$ ) as compared to $17.8 \%$ in C-section group. Neonatal morbidity was $35.5 \%$ in vaginal delivery as compared to $17.8 \%$ with C-section ( $p=0.012$ ) RR 2.627,95\% CI 1.216 to 5.678 . Prolonged hospitilisation was needed in $35.6 \%$ in vaginal delivery as compared to $15.6 \%$ in C-section group ( $\mathrm{p}=0.005) \mathrm{RR}=2.962,95 \%$ CI 1.354 to 6.478 Maternal morbidity in vaginal delivery group was $11.1 \%$ as against $7.8 \%$ in $\mathrm{C}$-section group and it was statistically not significant ( $p=0.54$ RR $=1.39,95 \%$ CI $0.447-4.307$ ). Neonatal morbidity comprised of perinatal asphyxia, seizures, DIC, hypoglycaemia, jaundice, meconium aspiration syndrome and respiratory distress. Immediate maternal morbidity consisted of wound infection, systemic infection, post-partum haemorrhage and postpartum depression .Delayed morbidity included irregular menstrual cycles and pelvic floor dysfunction.

Chart 2 shows that there were $30(33.3 \%)$ elective C-section and $60(66.6 \%)$ emergency C-sections. NICU admissions were more in elective C-section group (33.3\%) as compared to emergency C-section (11.7\%) and it was statistically significant ( $p=0.013) \mathrm{RR}=2.86,95 \% \mathrm{CI}$ 1.21 to 6.76. Neonatal morbidity was significantly high ( $30 \%)$ in elective CS group as compared to emergency CS $11.7 \% \quad(\mathrm{p}=0.03) \quad \mathrm{RR}=2.57,95 \%$ CI 1.06 to 6.23 . However Maternal morbidity was not associated with type of CS $p=0.25$; RR $=1.39,95 \% \mathrm{CI}$ 0.447 to 4.307 
Table 2 shows the follow up of breech deliveries for 1-2 years. 93.3\% of infants delivered by $\mathrm{C}$-section and $91.1 \%$ of those who delivered vaginally had no morbidity at $1-2$ years while $3.3 \%$ of infants in the C-section group had morbidity which comprised of recurrent respiratory tract infection and delayed milestones and underweight. Early neonatal mortality in C-section and vaginal delivery group was $3.3 \%$ and $8.8 \%$ respectively and the cause was perinatal asphyxia. The lone late neonatal mortality was due to Hypoxic ischaemic encephalopathy in C-section group. Infant mortality was $5.5 \%$ and $8.8 \%$ in C-section and vaginal delivery group respectively and cause was encephalopathy. There was no significant morbidity for mother in either group.

DISCUSSION: Vaginal breech birth can be associated with a higher risk of perinatal mortality and short-term neonatal morbidity than elective Caesarean section and our study corresponds with study by Hofmeyr M, Hannah M etal (2003) ${ }^{2}$.However careful case selection and labour management in a modern obstetrical setting may achieve a level of safety similar to elective caesarean section. With careful case selection and labour management, perinatal mortality occurs in approximately 2 per 1000 births and serious short-term neonatal morbidity in approximately $2 \%$ of breech infants 4 . Many recent retrospective and prospective reports of vaginal breech delivery that follow specific protocols have noted excellent neonatal outcomes 2 . Our series had a neonatal mortality rate of $8.8 \%$ Long-term neurological infant outcomes do not differ by planned mode of delivery even in the presence of serious short-term neonatal morbidity. Planned vaginal delivery of a term singleton breech fetus may be reasonable under hospital-specific protocol guidelines for both eligibility and labor management. Before a vaginal breech delivery is planned, women should be informed that the risk of perinatal or neonatal mortality or short-term serious neonatal morbidity may be higher than if a cesarean delivery is planned, and the patient's informed consent should be documented 5

CONCLUSION: The probability that vaginal breech birth can be associated with a higher risk of perinatal mortality and short-term neonatal morbidity than elective Caesarean section should be kept in mind. However careful case selection and labour management in a modern obstetrical setting may achieve a level of safety similar to elective Caesarean section. Long-term neurological infant outcomes do not differ by planned mode of delivery even in the presence of serious short-term neonatal morbidity.

\section{REFERENCES}

1. HannahME,HannahWJ,HewsonSA,HodnettED,SaigalS,WillanAR.PlannedCSvsplannedvagi nal birth for breech presentation at term: a randomized multicenter trial, Term Breech Trial Collaborative Group.Lancet 2000:356:1375-1383

2. HofmeyrGJ,HannahM,LawrieTA.Planned caesarean section for term breech delivery:RHL commentary(last revised:8 September 2003).The WHO Reproductive Health Library;Geneva:WHO

3. EnabudosoEJ,Gharoro EP(2005):Current use of Apgar score in the labour ward for resuscitation and referral of newbornbabies.Trop J Obst Gynaecol.22(1):46-49

4. IgwegbeA.O,MonagoE.N,UgboajaJ.O.Caesareanvs vaginal delivery for term breech presentation:A comparative analysis.Afr.J.Biomed. Res.(January 2010)15-18

5. ACOG Committee opinion, number 340,july 2006 
TABLE - 1

\begin{tabular}{|c|c|c|}
\hline & $\begin{array}{l}\text { Caesarean delivery } \\
\qquad n=90\end{array}$ & $\begin{array}{l}\text { Vaginal delivery } \\
\quad n=45\end{array}$ \\
\hline $\begin{array}{ll}\text { MATERNAL AGE } & \text { (Years) } \\
\text { - } & 19-25 \\
- & 26-30 \\
- & >30\end{array}$ & $\begin{array}{ll}56 & (62.2 \%) \\
25 & (27.7 \%) \\
9 & (10 \%)\end{array}$ & $\begin{array}{ll}29 & (64.4 \%) \\
14 & (31.1) \\
2 & (4.4 \%)\end{array}$ \\
\hline $\begin{aligned} \text { PARITY } \\
\begin{aligned}- & 0 \\
- & 1-2\end{aligned}\end{aligned}$ & $\begin{array}{ll}65 & (72.2 \%) \\
25 & (27.8 \%)\end{array}$ & $\begin{array}{ll}25 & (62.2 \%) \\
17 & (37.8 \%)\end{array}$ \\
\hline $\begin{aligned} \text { GESTATIONAL AGE(WKS) } \\
\begin{aligned} \text { - } & 36-37 \\
\text { - } & 37-40 \\
\text { - } & >40\end{aligned}\end{aligned}$ & $\begin{array}{ll}16 & (17.8 \%) \\
70 & (77.8 \%) \\
4 & (4.4 \%)\end{array}$ & $\begin{array}{ll}11 & (24.4 \%) \\
10 & (66.7 \%) \\
4 & (8.9 \%)\end{array}$ \\
\hline $\begin{array}{cl}\text { CS } & \text { DELIVERY } \\
& \text { ELECTIVE } \\
& \text { E EMERGENCY }\end{array}$ & $\begin{array}{ll}30 & (33.3 \%) \\
60 & (66.6 \%)\end{array}$ & 45 \\
\hline $\begin{array}{l}\text { BIRTH WEIGHT } \\
\begin{aligned} \text { - } & <1500 \\
\text { - } & 1500-3500 \\
\text { - } & >3500\end{aligned}\end{array}$ & $\begin{array}{ll}3 & (3.3 \%) \\
80 & (88.9 \%) \\
7 & (7.8 \%)\end{array}$ & $\begin{array}{ll}3 & (6.7 \%) \\
& 42 \quad(93.3 \%) \\
0 & \end{array}$ \\
\hline
\end{tabular}

TABLE - 2

\begin{tabular}{|c|c|c|c|c|c|}
\hline $\begin{array}{l}\text { Follow up } \\
1-2 \text { years }\end{array}$ & $\begin{array}{l}\text { C-section } \\
n=90\end{array}$ & $\begin{array}{l}\text { Vaginal } \\
n=45\end{array}$ & RR & 95\%CI for RR & $\mathrm{p}$ value \\
\hline $\begin{array}{c}\text { Infant Morbidity } \\
\text { - } \text { No } \\
\text { - } \quad \text { Yes }\end{array}$ & $\begin{array}{l}86(93.3 \%) \\
3(3.3 \%)\end{array}$ & $\begin{array}{l}41(91.1 \%) \\
0\end{array}$ & & & \\
\hline $\begin{array}{l}\text { Neonatal Mortality } \\
\text { - Early } \\
\text { - Late }\end{array}$ & $\begin{array}{l}3((3.3 \%) \\
1(1.1 \%)\end{array}$ & $\begin{array}{l}4(8.8 \%) \\
0\end{array}$ & $\begin{array}{l}2.67 \\
-\end{array}$ & $0.62-11.41$ & $\begin{array}{l}P=0.167 \\
P=0.667\end{array}$ \\
\hline Infant mortality & $5(5.5 \% \%)$ & $4(8.8 \%)$ & 1.6 & $0.45-5.67$ & $\mathrm{P}=0.346$ \\
\hline
\end{tabular}

Cause was encephalopathy.

There was no significant morbidity for mother in either group. 


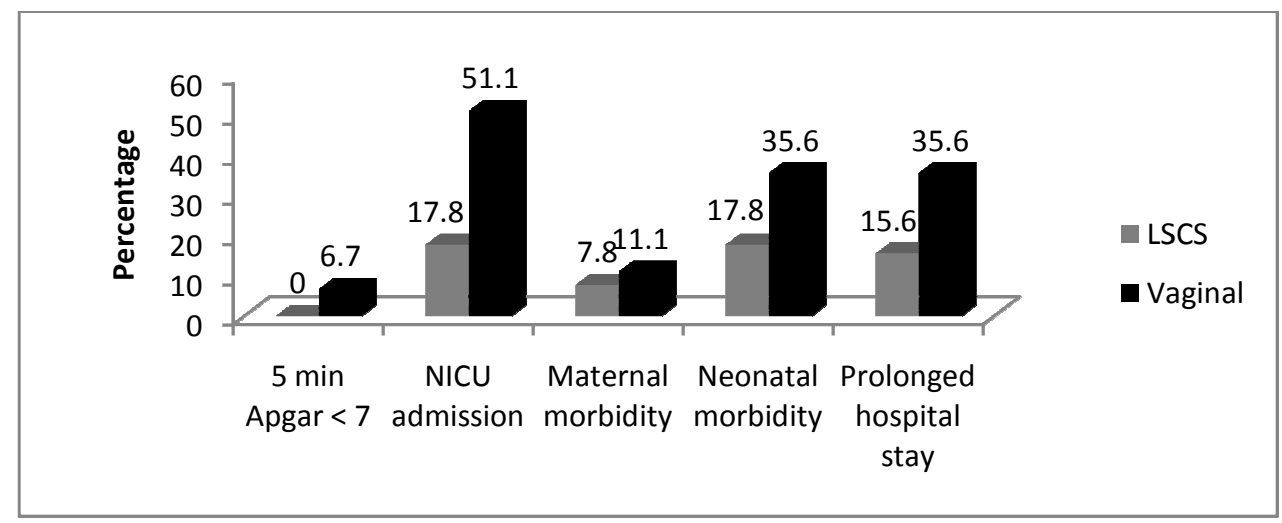

Chart 1: Perinatal outcome of babies following C-section and vaginal delivery for breech presentation

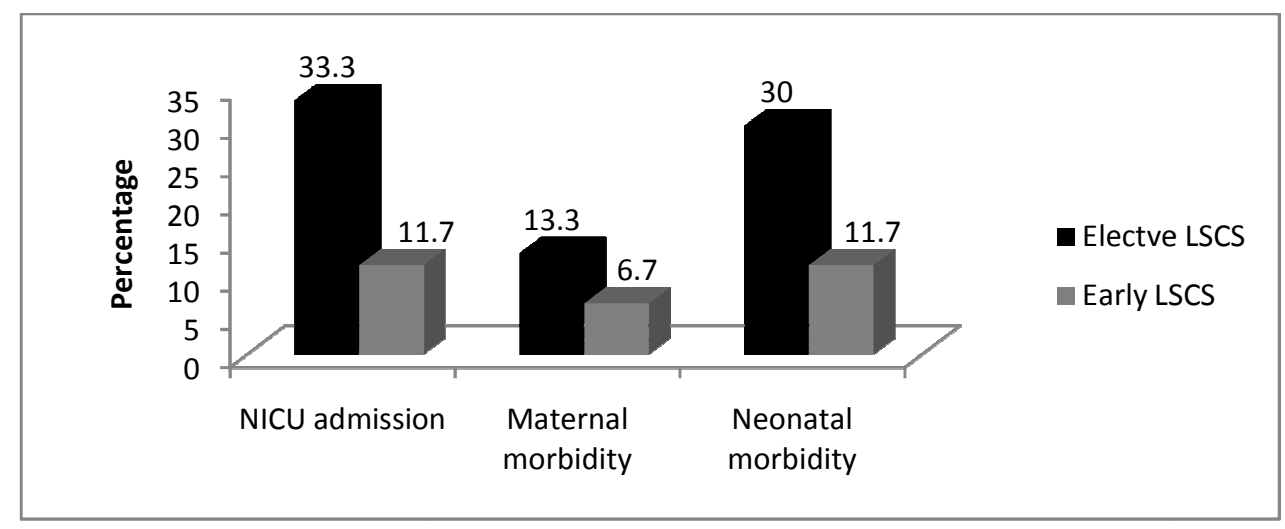

CHART 2 